\title{
Proteomic evaluation of genetically modified crops: current status and challenges
}

\author{
Chun Yan Gong and Tai Wang* \\ Key Laboratory of Plant Molecular Physiology, Institute of Botany, Chinese Academy of Sciences, Beijing, China
}

\section{Edited by:}

Setsuko Komatsu, National Institute of Crop Science, Japan

\section{Reviewed by:}

Mohammad-Zaman Nouri,

Rice Research Institute of Iran, Iran

Kentaro Kawaguchi, National

Agriculture and Food Research

Organization, Japan

*Correspondence:

Tai Wang, Key Laboratory of Plant Molecular Physiology, Institute of

Botany, Chinese Academy of

Sciences, 20 Nanxincun, Xiangshan,

Haidianqu, Beijing 100093, China.

e-mail: twang@ibcas.ac.cn
Hectares of genetically modified (GM) crops have increased exponentially since 1996, when such crops began to be commercialized. GM biotechnology, together with conventional breeding, has become the main approach to improving agronomic traits of crops. However, people are concerned about the safety of GM crops, especially GM-derived food and feed. Many efforts have been made to evaluate the unintended effects caused by the introduction of exogenous genes. "Omics" techniques have advantages over targeted analysis in evaluating such crops because of their use of high-throughput screening. Proteins are key players in gene function and are directly involved in metabolism and cellular development or have roles as toxins, antinutrients, or allergens, which are essential for human health. Thus, proteomics can be expected to become one of the most useful tools in safety assessment. This review assesses the potential of proteomics in evaluating various GM crops. We further describe the challenges in ensuring homogeneity and sensitivity in detection techniques.

Keywords: genetically modified crops, biological safety, unintended effects, substantial equivalence, proteome and proteomics

\section{INTRODUCTION}

Genetic modification has become the fastest-adopted technology in the history of modern agriculture. It involves the transfer of individual genes that encode specific desirable traits into the host, thus producing genetically modified (GM) crops (also transgenic crops) (Garcia-Canas et al., 2011). GM crops therefore possess improved agronomic traits, such as resistance to insects, tolerance to herbicides, improved productivity and quality, and other traits not present before genetic modification.

The hectares of GM crops continue to greatly increase worldwide (James, 2010; D’Alessandro and Zolla, 2011). Many important crops have been GM by transgenes; examples are maize (Zea mays), potato (Solanum tuberosum), rice (Oryza sativa), wheat (Triticum aestivum), soybean (Glycine max), tobacco (Nicotiana tabacum), tomato (Solanum lycopersicum), and cotton (Gossypium hirsutum). However, genetic modifications generally represent a double-edged sword. Besides inducing desired traits, modifications in a plant genome might result in unintended effects, which may affect human health or the environment (Ioset et al., 2007). Some unintended effects may result from integration of the transgene and/or biological interactions caused by transgene-encoding proteins, which can be predicted by knowledge of transgene integration sites, transgene function and transgene-related metabolic pathways. However, transgene integration and/or transformation and tissue culture during transgenic progress may induce unintended genomic alterations in GM plants such as deletions, insertions, and rearrangements, which may generate secondary or pleiotropic effects (Kuiper et al., 2001; Cellini et al., 2004; Garcia-Canas et al., 2011). The unintended effects associated with genomic alterations are unpredictable (Garcia-Canas et al., 2011). With the commercialization of GM crops, these unintended effects are one of the most controversial issues in debating the biological safety of GM crops. A systematic comparative analysis of molecular features of GM crops and their comparators is needed to clarify unintended effects (Cellini et al., 2004; Garcia-Canas et al., 2011).

The concept of "substantial equivalence" was proposed as a cornerstone of biological safety assessment in many countries (OECD, 1993). Although "the principle left much scope for individual (and national) interpretation" (Kok and Kuiper, 2003), it is still an acceptable standard to evaluate the biological safety of GM crops. The increasingly use of "omics" technologies, including genomics, proteomics, and metabolomics, in GM crops analysis has provided important information on the molecular characteristics of GM crops and extended our understanding of the biological safety of GM crops. In this mini-review, we briefly discuss technologies used in evaluating GM crops, and summarize current proteomics insights into GM crops.

\section{TECHNOLOGIES FOR EVALUATING GM CROPS}

Targeted analysis is the primary method used for evaluating GM crops. According to the Organisation for Economic Co-operation and Development (OECD, 2006), 50-100 or more analytes are selected for targeted analysis of each crop variety. These analytes typically represent a limited number of crop compositions; they cannot cover unknown toxins, antinutrients, or other secondary products that could result from the genetic modification (Kuiper et al., 2001). Accordingly, targeted analysis is useful for detecting primary or intended but not unintended effects of genetic modification and has been considered biased (Millstone et al., 1999).

Profiling techniques have emerged as useful approaches to evaluate the unintended effects. They allow for simultaneous 
characterization and comparison of the genome, proteome, and metabolome of an organism, thus increasing the chances of detecting the unintended effects (Kuiper et al., 2003; Ruebelt et al., 2006). These non-targeted approaches have become more comprehensive than targeted analysis in characterizing the composition and performance of GM crops and detecting the unintended effects.

Transcriptome profiling has been used to characterize several GM crops, including maize, barley and rice (Coll et al., 2008, 2009; Kogel et al., 2010; Montero et al., 2011). Transcriptome profiling could be used to investigate gene expression changes in GM crops and has potential to detect unintended effects. But changes in a transcriptome do not necessarily lead to changes in a proteome or metabolome, therefore do not necessarily predict changes in food composition and quality (Chassy, 2010), so transcriptomic profiling is limited in evaluating unintended effects.

Besides transcriptomic techniques, proteomic and metabolomic methods are two complementary tools for evaluating GM crops. In recent years, metabolomic analysis has become prevalent (Catchpole et al., 2005; Baker et al., 2006; Garcia-Villalba et al., 2008; Kim et al., 2009). Because of its ability for use in analyzing a great number of metabolites and reducing the cost of analysis for each analyte, metabolomics has been considered a replacement for conventional compositional analysis (Chassy, 2010). However, this method is only capable of measuring hundreds of metabolites, not the thousands of metabolites in a plant (Davies, 2010). Furthermore, differences in metabolomic methodologies, data analysis, and statistical analysis have resulted in less reproducibility. Therefore, metabolomics may not be advantageous for safety assessment (Chassy, 2010). Proteins are key players in gene function and are directly involved in metabolism and cellular development, thus forming the central bridge between the transcriptome and metabolome (Salekdeh and Komatsu, 2007). Furthermore, proteins have roles as toxins, antinutrients, or allergens, which have great impact on human health. Therefore, proteomic studies would provide important information for understanding changes in biological processes after genetic modification and are important for evaluating biological safety of GM crops.

\section{PROTEOMIC CHARACTERISTICS OF GM CROPS}

2-D gel electrophoresis (2-DE) analysis, established in 1975, revolutionized the study of proteins (Klose, 1975; O'Farrell, 1975) and has helped in the development of proteomics. With use of immobilized pH gradients (Bjellqvist et al., 1982) and mass spectrometry (MS), proteomics has played important roles in understanding the mechanisms and protein-protein interaction networks underlying biological processes. Comparative proteomic strategies combined with 2-DE and MS and with liquid chromatography tandem mass spectrometry (LC-MS/MS) have been extensively used to evaluate the effects of genetic modification on the proteomes of eight GM crops: maize, pea, potato, rice, soybean, tobacco, tomato, and wheat (Table 1). These studies involved safety evaluation of GM crops and functional characterization of GM crops. Some studies investigated different varieties for detecting natural variation. Proteomic evaluation of a GM pea cultivar carrying $\alpha$-amylase inhibitor-1 ( $\alpha \mathrm{AI} 1)$ was discussed elsewhere (Chen et al., 2009; Islam et al., 2009; Ricroch et al., 2011). An excellent review discussed MS-based identification of transgenic proteins in GM crops (Garcia-Canas et al., 2011). So here we mainly discuss the recent status of proteomic evaluation of the other seven GM crops.

\section{MAIZE}

GM maize is the second most commercialized GM crop worldwide and has been approved for release in many countries (Coll et al., 2008). MON810 is insect-resistant GM maize produced by inserting a truncated form of the $c r y 1 A b$ gene from Bacillus thuringiensis in the maize genome. The proteomes of MON810 varieties and their comparators have been well studied because of their commercial importance (Albo et al., 2007; Zolla et al., 2008; Barros et al., 2010; Coll et al., 2011). Albo et al. (2007) compared the seed proteomes of MON810 variety BT (derived by crossing La73-Bt and La17-Bt) and the near-isogenic control (derived by crossing La73 and La17) and detected differences in six proteins: glucose and ribitol dehydrogenase appeared specific to BT, and endochitinase A to the control, with a difference in expression of triosephosphate isomerase 1, globulin$1 \mathrm{~S}$, cytosolic 3-phosphoglycerate kinase, and aldose reductase between BT and the control. Coll et al. (2011) found a few spots with 1.1- to 1.8-fold change in expression in seed proteomes of two other sets of MON810 varieties, PR33P67, and DKC6575, and their corresponding non-GM controls, PR33P66, and Tietar. The two studies showed that the proteomes of these GM varieties should be virtually identical to that of their comparators. However, Zolla et al. (2008) found that proteomes of seeds from PR33P67 and PR33P66 had 43 differentially expressed proteins (DEPs) possibly caused by the transgene. This situation was probably resulted from different cultured conditions of materials (agriculture field vs. environmentally controlled growth chamber). Barros et al. (2010) evaluated the effect of growing season, growing location, and transgenes on transcriptomes, proteomes, and metabolomes of maize seeds using the GM lines DKC78-15B (hybrid of event MON810) and DKC78-35R (hybrid of event NK603) and the near-isogenic non-GM hybrid variety CRN3505. Transgenes produced less variation in transcript, protein, or metabolite profiles of each sample pair than did environmental factors. Balsamo et al. (2011) compared leaf proteomes of four MON810 varieties and their controls using the same 2-DE-based proteomics and revealed seven DEPs in the DKBYG240-DKB240 pair, five in the DKBYG350-DKB350 pair, and none in the other two GM-non-GM pairs. Thus, the leaf proteomes of four MON810 varieties may have been similar to those of their non-GM counterparts. Together, these data suggested that the expression of transgenes in host maize plants had no significant effects on proteome of the host, and evaluating the molecular characteristics of GM crops should involve environmental factors.

\section{POTATO}

Potato is the fourth major crop widely grown worldwide. Despite diverse potato varieties with desired traits, GM potato is still 


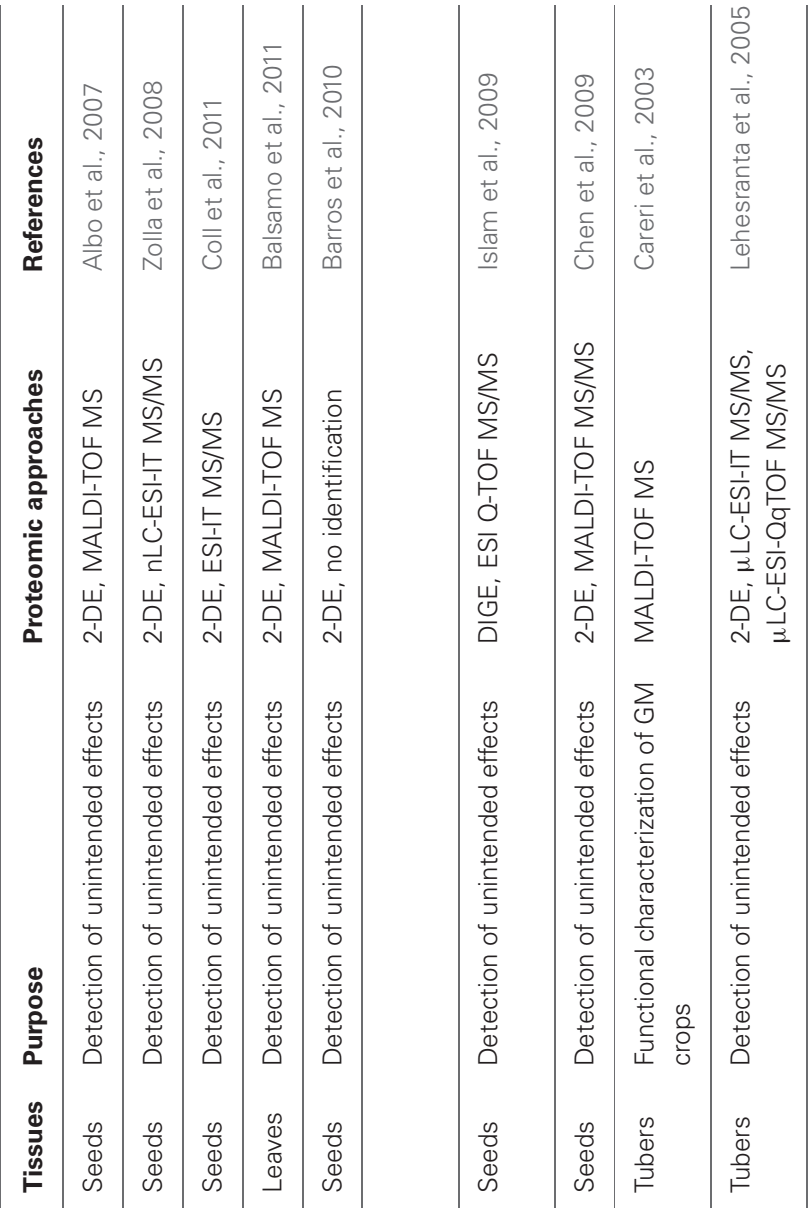

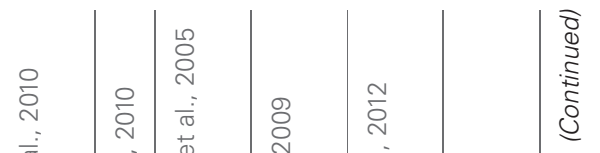

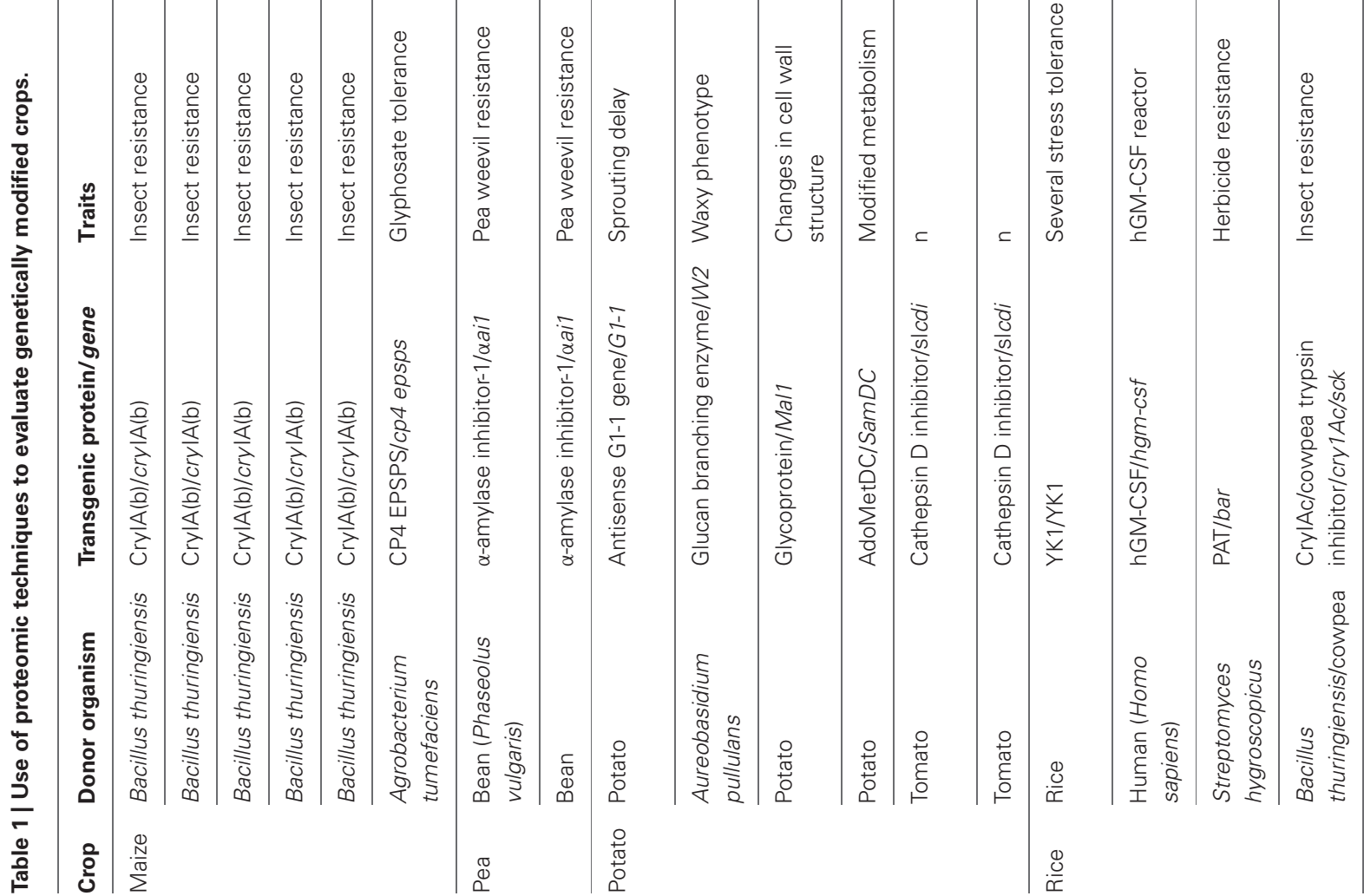




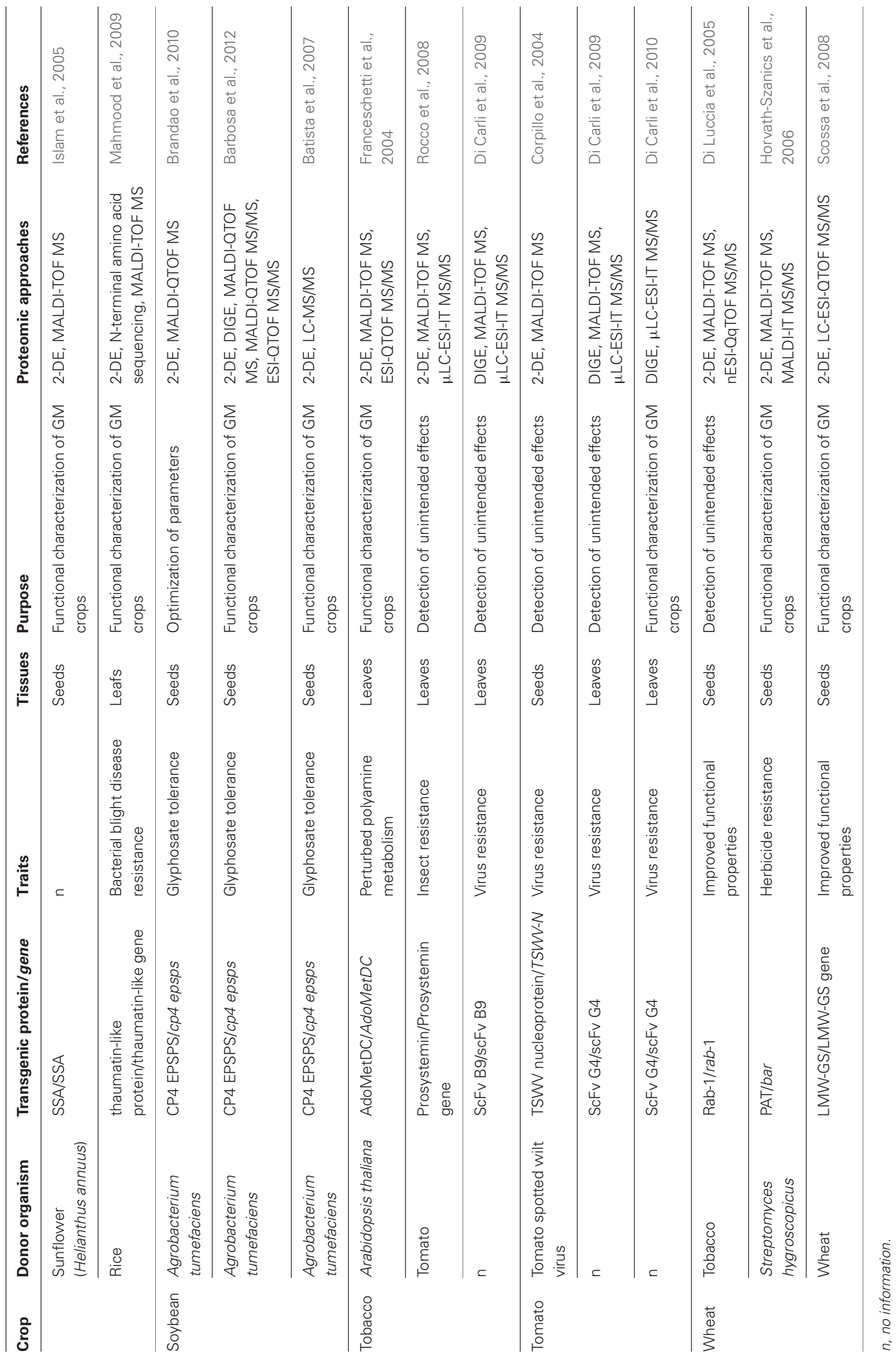


an important topic in potato breeding. Comparative proteomics was used to evaluate variation in tuber proteomes of a large collection of potato genotypes, including different varieties, landraces, and GM lines (Lehesranta et al., 2005). Different varieties and landraces showed clear separations, which indicates extensive genotypic variation; but the differences between GM and non-GM lines were less significant (Lehesranta et al., 2005). A proteomic study by Careri et al. (2003) showed that spatial sites of transgene-encoding proteins affected the proteome of the analyzed sample-GM potato PG50 carrying the antisense G1-1 gene of unknown function. Phenotypic and expression analysis confirmed that G1-1 was highly expressed in apical eyes, and its expression regulated transition from dormancy to sprouting tubers (Agrimonti et al., 2000; Marmiroli et al., 2000). A proteomic analysis revealed no difference between PG50 and nonGM lines with use of whole tubers but several differences with use of only apical eyes. This finding was consistent with the expression of G1-1 gene in apical eyes. Other studies found inconsistent proteome changes in different tissues of GM potato (Goulet et al., 2010; Khalf et al., 2010). Transgenic potato expressing cathepsin D inhibitor (S/CDI) showed significant variation in leaf proteome as compared with the control (Goulet et al., 2010) but no quantitative proteome difference in tubers of the two samples (Khalf et al., 2010). Because antisense G1-1 in PG50 affects only the proteome of tissue expressing antisense G1-1, the different effect of the slcdi transgene on leaf and tuber proteomes may result from different expression of the gene in leaves and tubers, although the two studies did not explore the different expression. Therefore, examining expression patterns and levels of transgenes is important to explain proteomic data.

\section{RICE}

Rice feeds one-quarter of world's population and is one of main crops in the world (Agrawal and Rakwal, 2011). It has become model system to study biological issues. GM rice has been quickly developed although commercialization of GM rice is still in debate. Gong et al. (2012) evaluated proteome differences in seeds from two sets of GM rice (Bar68-1 carrying bar and 2036-1a carrying $c r y 1 A c / s c k$ ) and their controls by 2-DE differential ingel electrophoresis (2D-DIGE). To obtain relatively objective data, this study included other rice varieties to evaluate proteome variations related to spontaneous genetic variation, genetic breeding, and genetic modifications. GM events did not substantially alter protein profiles as compared with conventional genetic breeding and natural genetic variation (Gong et al., 2012). However, use of GM rice expressing human granulocyte-macrophage colony stimulation factor and shotgun analysis of trypsin digests labeled by isobaric tags for relative and absolute quantitation (iTRAQ) revealed more DEPs (103 proteins) between GM rice and wildtype rice endosperm (Luo et al., 2009). No parallel experiments have compared the output of the two proteomic strategies with the same set of GM rice and control, so explaining these differences is difficult. One explanation may be that the LC-MS/MS shotgun approach provides more comprehensive identification of proteins. In addition, different exogenous genes and/or different insert events of these genes in host genomes may have different impacts on the host crops. Indeed, several proteomic studies of
GM rice have investigated biological changes with exogenous gene insertion in seeds (Islam et al., 2005), cultured cells (Takahashi et al., 2005), and leaf blades (Mahmood et al., 2009). A final explanation may be that these transgene-encoding proteins accumulate at different levels in analyzed tissues, for a differential effect on proteome profiling of the analyzed tissues, as we discussed previously for GM potato. Therefore, the safety assessment of GM crops should be case by case.

\section{SOYBEAN}

Soybean is one of the main foods responsible for allergic reactions worldwide, but GM soybean remains one of the most commercialized GM crop. Batista et al. (2007) compared seed proteome of Roundup Ready soybean carrying cp4 epsps and nontransgenic control; they found that soybean endogenous allergen expression did not seem to be altered after genetic modification. Brandao et al. (2010) evaluated variables that affect the analysis of protein profiles before comparative proteomic analysis of Roundup Ready soybean (MSOY7575RR) and a non-GM comparator (MSOY7501). Optimal parameters were manual image editing, 300- $\mu$ g protein loading for $3-10 \mathrm{pH}$ range strips and 500- $\mu$ g loading for 4-7 pH strips. The authors identified 10 DEPs between seed proteomes via 2-DE with both $\mathrm{pH} 3-10$ and 4-7 gel strips. Barbosa et al. (2012) used a more sensitive 2D-DIGE technique to further compare protein profiles of seeds from the same sample pair and revealed only four DEPs with $\mathrm{pH} 4-7$ strips, of which only one DEP was identical to that identified by Brandao et al. (2010). The two studies used the same materials and protein extraction methods, so the inconsistent results probably derived from standards for judging DEPs in comparative proteomics. Barbosa et al. (2012) identified DEPs based on statistical analysis ( $p \leq 0.05$, by Student $t$-test) and fold change in expression ( $\geq 1.5,50 \%$ variation), but Brandao et al. (2010) identified DEPs by only fold change in expression $(\geq 1.8, \sim 90 \%$ variation). Therefore, statistical analysis may be important to determine differential expression of proteins.

\section{TOBACCO}

Tobacco is of great economic importance, despite hazards and environmental problems accompanying its production. Di Carli et al. (2009) used 2D-DIGE to compare the leaf proteomes of GM tobacco expressing ScFv (B9) antibody against the G1 envelope glycoprotein of tomato spotted wilt virus (TSWV) and a non-GM control. The recombinant antibody did not significantly affect leaf protein profiles. However, a proteomic study of tobacco expressing tomato prosystemin showed that expression of the gene greatly changed leaf protein profiles of the host (Rocco et al., 2008). The two studies also indicated the need for evaluating substantial equivalence in GM plants on a case-by-case basis. Furthermore, changes in host proteome are associated with the expression level of a transgene, as shown in the study of GM tobacco lines overexpressing S-adenosylmethionine decarboxylase (AdoMetDC) (Franceschetti et al., 2004). A comparison of leaf proteomes from three GM tobacco lines with different levels of AdoMetDC revealed that as compared with the control, the proteome of the GM line with the highest level of AdoMetDC had the largest numbers of DEPs and that 
of the line with the lowest level of AdoMetD had the lowest numbers of DEPs.

\section{TOMATO}

Tomato has great economic and nutritional value. Genetic modifications of tomato mainly involved virus resistance, and delayed fruit ripening. Corpillo et al. (2004) first assessed the substantial equivalence of GM tomato using proteomic approaches. The authors found no qualitative or quantitative differences between the GM tomato that was GM for resistance to TSWV and the non-GM control. Similarly, Di Carli et al. (2009) demonstrated that expression of $\operatorname{scFv}(\mathrm{G} 4)$ against the CMV coat protein in tomato did not cause pleiotropic effects. Di Carli et al. (2010) further evaluated protein profiles of the same $\mathrm{scFv}(\mathrm{G} 4)$-expressed GM tomato and the wild-type after cucumber mosaic virus (CMV) infection. Proteomic data showed that proteins related to photosynthesis, photorespiration, carbon metabolism, and defense mechanism were downregulated by CMV in infected leaves, which highlighted the mechanisms of the plant-virus interactions.

\section{WHEAT}

Wheat is the second most important cereal crop in the world and constitutes a major part of the human diet (Salekdeh and Komatsu, 2007). Currently, GM wheat mainly involves improvement in protein quality. A proteomic study of GM bread wheat overexpressing a low-molecular-weight glutenin subunit (LMW-GS) revealed a series of variations, including overaccumulation of the LMW glutenin and downregulation of all other classes of storage proteins, which constituted a compensatory mechanism (Scossa et al., 2008). The proteomic analysis of two other GM durum wheat, Svevo B730 1-1, expressing the wild-type form of tobacco $r a b$-1, and Ofanto B688 1-2, expressing a mutated form of $r a b-1$, and their control lines (Svevo and Ofanto) revealed inconsistent results (Di Luccia et al., 2005). Tobacco rab-1 influences the trafficking of gluten proteins through the secretory system by up- or downregulating the transport step from the endoplasmic reticulum to the Golgi apparatus. Proteomic data showed significant differences between Ofanto B688 1-2, with downregulated trafficking, and the Ofanto

\section{REFERENCES}

Agrawal, G. K., and Rakwal, R. (2011). Rice proteomics: a move toward expanded proteome coverage to comparative and functional proteomics uncovers the mysteries of rice and plant biology. Proteomics 11, 1630-1649.

Agrimonti, C., Visioli, G., and Marmiroli, N. (2000). In vitro and in silico analysis of two genes (A2-1 and G1-1) differentially regulated during dormancy and sprouting in potato tubers. Potato Res. 43, 325-333.

Albo, A. G., Mila, S., Digilio, G., Motto, M., Aime, S., and Corpillo, D. (2007). Proteomic analysis of a genetically modified maize flour carrying CrylAb gene and comparison to the corresponding wild-type. Maydica 52, 443-455.

Baker, J. M., Hawkins, N. D., Ward, J. L., Lovegrove, A., Napier, J. A., Shewry, P. R., et al. (2006). A metabolomic study of substantial equivalence of field-grown genetically modi381-392.

Balsamo, G. M., Cangahuala-Inocente, G. C., Bertoldo, J. B., Terenzi, H., and Arisi, A. C. M. (2011). Proteomic analysis of four Brazilian MON810 maize varieties and their four non-genetically-modified isogenic varieties. J. Agric. Food Chem. 59, 11553-11559. fied wheat. Plant Biotechnol. J. 4,

control but none between Svevo B730 1-1, with upregulated trafficking, and the Svevo control. The study concluded that the proteomic approach is powerful for investigating protein changes in GM crops and for understanding events involved in quality trait. Indeed, Horvath-Szanics et al. (2006) used proteomic methods to identify stress-induced proteins in herbicide-resistant GM wheat lines and found changed level of LMW seed proteins and sensitivity to drought stress in this GM wheat under drought stress.

\section{FUTURE AND CHALLENGES}

Proteomics has been used to detect unintended effects caused by genetic modification in GM crops. Results from most studies involving comparative proteomics demonstrated essentially identical protein profiles in GM crops and controls. Furthermore, genetic modification caused less variation in GM crops than the natural variability derived from conventional breeding and genotypic variation. However, some other studies demonstrated that genetic modification affected the proteomes of recipient crops. The discrepancy may be explained by differences in (1) the inserted exogenous genes, (2) the insertion sites of exogenous genes, (3) the expression levels of exogenous genes in recipient crops, (4) the specific organs used for study, (5) GM crop planting conditions, and (6) detection techniques. Therefore, proteomic evaluation of GM crops should be considered on a case-by-case basis.

However, proteomic approaches need improvement. First, homogeneity is needed in experimental quality and data handling (Lay et al., 2006). Experiments should involve as many biological repeats as possible. Statistical analysis and biological validation are also needed. These requirements are applicable to all "omics" techniques. Second, different methods should be combined, including different combinations of "omics" as well as the combination of "omics" and targeted analysis (Ricroch et al., 2011). More sensitive techniques are needed for detecting a larger range of unintended effects.

\section{ACKNOWLEDGMENTS}

This work was supported by a grant from the Chinese Ministry of Agriculture (grant no. 2011ZX08012-002).

Barbosa, H. S., Arruda, S. C., Azevedo, R. A., and Arruda, M. A. (2012). New insights on proteomics of transgenic soybean seeds: evaluation of differential expressions of enzymes and proteins. Anal. Bioanal. Chem. 402, 299-314.

Barros, E., Lezar, S., Anttonen, M. J., Van Dijk, J. P., Rohlig, R. M., Kok, E. J., et al. (2010). Comparison of two GM maize varieties with a near-isogenic non-GM variety using transcriptomics, proteomics and metabolomics. Plant Biotechnol. J. 8, 436-451.

Batista, R., Martins, I., Jenö, P., Ricardo, C. P., and Oliveira, M. M. (2007). A proteomic study to identify soya allergens - the human response to transgenic versus non-transgenic soya samples. Int. Arch. Allergy Immunol. 144, 29-38.

Bjellqvist, B., Ek, K., Righetti, P. G., Gianazza, E., Gorg, A., Westermeier, R., et al. (1982). Isoelectric-focusing in immobilized $\mathrm{pH}$ gradients principle, methodology and some applications. J. Biochem. Biophys. Methods 6, 317-339.

Brandao, A. R., Barbosa, H. S., and Arruda, M. A. (2010). Image analysis of two-dimensional gel electrophoresis for comparative proteomics of transgenic and non-transgenic soybean seeds. J. Proteomics 73, 1433-1440.

Careri, M., Elviri, L., Mangia, A., Zagnoni, I., Agrimonti, C., Visioli, 
G., et al. (2003). Analysis of protein profiles of genetically modified potato tubers by matrix-assisted laser desorption/ionization timeof-flight mass spectrometry. Rapid Commun. Mass Spectrom. 17, 479-483.

Catchpole, G. S., Beckmann, M., Enot, D. P., Mondhe, M., Zywicki, B., Taylor, J., et al. (2005). Hierarchical metabolomics demonstrates substantial compositional similarity between genetically modified and conventional potato crops. Proc. Natl. Acad. Sci. U.S.A. 102, 14458-14462.

Cellini, F., Chesson, A., Colquhoun, I., Constable, A., Davies, H. V., Engel, K. H., et al. (2004). Unintended effects and their detection in genetically modified crops. Food Chem. Toxicol. 42, 1089-1125.

Chassy, B. M. (2010). Can -omics inform a food safety assessment? Regul. Toxicol. Pharmacol. 58, S62-S70.

Chen, H. C., Bodulovic, G., Hall, P. J., Moore, A., Higgins, T. J. V., Djordjevic, M. A., et al. (2009). Unintended changes in protein expression revealed by proteomic analysis of seeds from transgenic pea expressing a bean alphaamylase inhibitor gene. Proteomics 9, 4406-4415.

Coll, A., Nadal, A., Collado, R., Capellades, G., Messeguer, J., Melé, E., et al. (2009). Gene expression profiles of MON810 and comparable non-GM maize varieties cultured in the field are more similar than are those of conventional lines. Transgenic Res. 18, 801-808.

Coll, A., Nadal, A., Palaudelmàs, M., Messeguer, J., Melé, E., Puigdomènech, P., et al. (2008). Lack of repeatable differential expression patterns between MON810 and comparable commercial varieties of maize. Plant Mol. Biol. 68, 105-117.

Coll, A., Nadal, A., Rossignol, M., Puigdomènech, P., and Pla, M. (2011). Proteomic analysis of MON810 and comparable non-GM maize varieties grown in agricultural fields. Transgenic Res. 20, 939-949.

Corpillo, D., Gardini, G., Vaira, A. M., Basso, M., Aime, S., Accotto, G. R., et al. (2004). Proteomics as a tool to improve investigation of substantial equivalence in genetically modified organisms: the case of a virus-resistant tomato. Proteomics 4, 193-200.

D'Alessandro, A., and Zolla, L. (2011). We are what we eat: food safety and proteomics. J. Proteome Res. 11, 26-36.

Davies, H. (2010). A role for "omics" technologies in food safety assessment. Food Control 21, 1601-1610.

Di Carli, M., Villani, M. E., Bianco, L., Lombardi, R., Perrotta, G., Benvenuto, E., et al. (2010). Proteomic analysis of the plantvirus interaction in cucumber mosaic virus (CMV) resistant transgenic tomato. J. Proteome Res. 9, 5684-5697.

Di Carli, M., Villani, M. E., Renzone, G., Nardi, L., Pasquo, A., Franconi, R., et al. (2009). Leaf proteome analysis of transgenic plants expressing antiviral antibodies. J. Proteome Res. 8, 838-848.

Di Luccia, A., Lamacchia, C., Fares, C., Padalin, L., Mamone, G., La Gatta, B., et al. (2005). A proteomic approach to study protein variation in GM durum wheat in relation to technological properties of semolina. Ann. Chim. 95, 405-414.

Franceschetti, M., Perry, B., Thompson, B., Hanfrey, C., and Michael, A. J. (2004). Expression proteomics identifies biochemical adaptations and defense responses in transgenic plants with perturbed polyamine metabolism. FEBS Lett. 576, 477-480.

Garcia-Canas, V., Simo, C., Leon, C., Ibanez, E., and Cifuentes, A. (2011). Ms-based analytical methodologies to characterize genetically modified crops. Mass Spectrom. Rev. 30, 396-416.

Garcia-Villalba, R., Leon, C., Dinelli, G., Segura-Carretero, A., Fernandez-Gutierrez, A., Garcia-Canas, V., et al. (2008). Comparative metabolomic study of transgenic versus conventional soybean using capillary electrophoresis-time-of-flight mass spectrometry. J. Chromatogr. A 1195, 164-173.

Gong, C. Y., Li, Q., Yu, H. T., Wang, Z., and Wang, T. (2012). Proteomics insight into the biological safety of transgenic modification of rice as compared with conventional genetic breeding and spontaneous genotypic variation. J. Proteome Res. 11, 3019-3029.

Goulet, C., Benchabane, M., Anguenot, R., Brunelle, F., Khalf, M., and Michaud, D. (2010). A companion protease inhibitor for the protection of cytosol-targeted recombinant proteins in plants. Plant Biotechnol. J. 8, 142-154.

Horvath-Szanics, E., Szabo, Z., Janaky, T., Pauk, J., and Hajos, G. (2006). Proteomics as an emergent tool for identification of stress-induced proteins in control and genetically modified wheat lines. Chromatographia 63 , S143-S147.

Ioset, J. R., Urbaniak, B., Ndjoko-Ioset, K., Wirth, J., Martin, F., Gruissem, W., et al. (2007). Flavonoid profiling among wild type and related GM wheat varieties. Plant Mol. Biol. 65, 645-654.

Islam, N., Campbell, P. M., Higgins, T. J. V., Hirano, H., and Akhurst, R. J. (2009). Transgenic peas expressing an alpha-amylase inhibitor gene from beans show altered expression and modification of endogenous proteins. Electrophoresis 30, 1863-1868.

Islam, N., Upadhyaya, N. M. Campbell, P. M., Akhurst, R., Hagan, N., and Higgins, T. J. V. (2005). Decreased accumulation of glutelin types in rice grains constitutively expressing a sunflower seed albumin gene. Phytochemistry 66, 2534-2539.

James, C. (2010). Global Status of Commercialized Biotech/GM Crops. 2010.ISAAA Brief No. 42. Ithaca, NY: ISAAA.

Khalf, M., Goulet, C., Vorster, J., Brunelle, F., Anguenot, R., Fliss, I. et al. (2010). Tubers from potato lines expressing a tomato Kunitz protease inhibitor are substantially equivalent to parental and transgenic controls. Plant Biotechnol. J. 8, 155-169.

Kim, H., Kim, S., Park, Y., Kwon, S., Liu, J., Joung, H., et al. (2009). Metabolic profiles of genetically modified potatoes using a combination of metabolite fingerprinting and multivariate analysis. Biotechnol. Bioprocess Eng. 14, 738-747.

Klose, J. (1975). Protein mapping by combined isoelectric focusing and electrophoresis of mouse tissuesnovel approach to testing for induced point mutations in mammals. Humangenetik 26, 231-243.

Kogel, K.-H., Voll, L. M., Schäfer, P., Jansen, C., Wu, Y., Langen, G., et al. (2010). Transcriptome and metabolome profiling of field-grown transgenic barley lack induced differences but show cultivar-specific variances. Proc. Natl. Acad. Sci. U.S.A. 107, 6198-6203.

Kok, E. J., and Kuiper, H. A. (2003). Comparative safety assessment for biotech crops. Trends Biotechnol. 21, 439-444.

Kuiper, H. A., Kleter, G. A., Noteborn, H. P. J. M., and Kok, E. J. (2001). Assessment of the food safety issues related to genetically modified foods. Plant J. 27, 503-528.

Kuiper, H. A., Kok, E. J., and Engel, K. H. (2003). Exploitation of molecular profiling techniques for GM food safety assessment. Curr. Opin. Biotechnol. 14, 238-243.

Lay, J. O., Borgmann, S., Liyanage, R., and Wilkins, C. L. (2006). Problems with the "omics". Trends Anal. Chem. 25, 1046-1056.

Lehesranta, S. J., Davies, H. V., Shepherd, L. V. T., Nunan, N., McNicol, J. W., Auriola, S., et al. (2005). Comparison of tuber proteomes of potato varieties, landraces, and genetically modified lines. Plant Physiol. 138, 1690-1699.

Luo, J., Ning, T., Sun, Y., Zhu, J., Zhu, Y., Lin, Q., et al. (2009). Proteomic analysis of rice endosperm cells in response to expression of hGM-CSF. J. Proteome Res. 8, 829-837.

Mahmood, T., Jan, A., and Komatsu, S. (2009). Proteomic analysis of bacterial blight defence signalling pathway using transgenic rice overexpressing thaumatin-like protein. Biol. Plant. 53, 285-293.

Marmiroli, N., Agrimonti, C., Visioli, G., Colauzzi, M., Guarda, G., and Zuppini, A. (2000). Silencing of G11 and A2-1 genes. Effects on general plant phenotype and on tuber dormancy in Solanum tuberosum L. Potato Res. 43, 313-323.

Millstone, E., Brunner, E., and Mayer, S. (1999). Beyond 'substantial equivalence'. Nature 401, 525-526.

Montero, M., Coll, A., Nadal, A., Messeguer, J., and Pla, M. (2011). Only half the transcriptomic differences between resistant genetically modified and conventional rice are associated with the transgene. Plant Biotechnol. J. 9, 693-702.

O'Farrell, P. H. (1975). High resolution two-dimensional electrophoresis of proteins. J. Biol. Chem. 250, 4007-4021.

Organisation for Economic Cooperation and Development. (1993). Safety Evaluation of Foods Derived by Modern Biotechnology: Concepts and Principles. Paris: Organisation for Economic Co-operation and Development.

Organisation for Economic Cooperation and Development. (2006). An Introduction to the Food/Feed Safety Consensus Documents of the Task Force. Series on the Safety of Novel Foods and Feeds, No 14. Paris: Organization for Economic Cooperation and Development.

Ricroch, A. E., Bergé, J. B., and Kuntz, M. (2011). Evaluation of genetically 
engineered crops using transcriptomic, proteomic, and metabolomic profiling techniques. Plant Physiol. 155, 1752-1761.

Rocco, M., Corrado, G., Arena, S., D’Ambrosio, C., Tortiglione, C., Sellaroli, S., et al. (2008). The expression of tomato prosystemin gene in tobacco plants highly affects host proteomic repertoire. J. Proteomics 71, 176-185.

Ruebelt, M. C., Lipp, M., Reynolds, T. L., Schmuke, J. J., Astwood, J. D., Dellapenna, D., et al. (2006). Application of two-dimensional gel electrophoresis to interrogate alterations in the proteome of gentically modified crops. 3. Assessing unintended effects. J. Agric. Food Chem. 54, 2169-2177.
Salekdeh, G. H., and Komatsu, S. (2007). Crop proteomics: aim at sustainable agriculture of tomorrow. Proteomics 7, 2976-2996.

Scossa, F., Laudencia-Chingcuanco, D., Anderson, O. D., Vensel, W. H., Lafiandra, D., D'Ovidio, R., et al. (2008). Comparative proteomic and transcriptional profiling of a bread wheat cultivar and its derived transgenic line overexpressing a low molecular weight glutenin subunit gene in the endosperm. Proteomics 8, 2948-2966.

Takahashi, H., Hotta, Y., Hayashi, M., Kawai-Yamada, M., Komatsu, S., and Uchimiya, H. (2005). High throughput metabolome and proteome analysis of transgenic rice plants (Oryza sativa L.). Plant Biotechnol. 22, 47-50.

Zolla, L., Rinalducci, S., Antonioli, P., and Righetti, P. G. (2008). Proteomics as a complementary tool for identifying unintended side effects occurring in transgenic maize seeds as a result of genetic modifications. J. Proteome Res. 7, 1850-1861.

Conflict of Interest Statement: The authors declare that the research was conducted in the absence of any commercial or financial relationships that could be construed as a potential conflict of interest.

Received: 28 November 2012; paper pending published: 04 January 2013, accepted: 19 February 2013; published online: 07 March 2013.

Citation: Gong CY and Wang T (2013)

Proteomic evaluation of genetically modified crops: current status and challenges. Front. Plant Sci. 4:41. doi: 10.3389/fpls. 2013.00041

This article was submitted to Frontiers in Plant Proteomics, a specialty of Frontiers in Plant Science.

Copyright (C) 2013 Gong and Wang. This is an open-access article distributed under the terms of the Creative Commons Attribution License, which permits use, distribution and reproduction in other forums, provided the original authors and source are credited and subject to any copyright notices concerning any third-party graphics etc. 